\title{
Does the Method of Vocabulary Presentation Make a Difference?
}

Adina Levine

Thea Reves

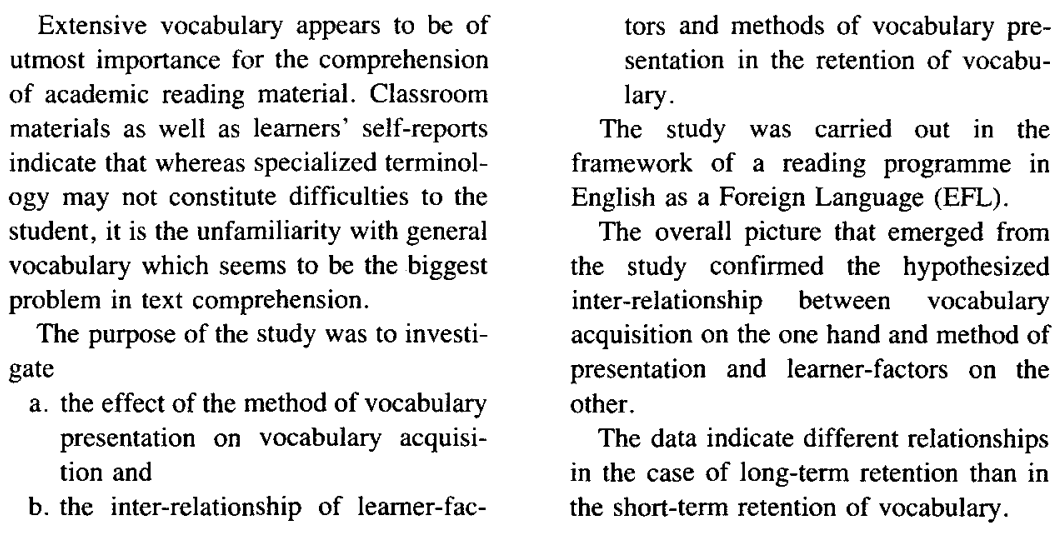

Lack of adequate vocabulary is often cited by EFL learners as one of the obstacles to text comprehension. Classroom experience as well as learners' self-reports indicate that it is easier for the reader of academic texts to cope with special terminology than with general vocabulary.

A widespread approach to vocabulary learning seems to be based on the belief that vocabulary acquisition is only a by-product of the instruction of reading. According to this view, exposing the student to a large amount of only partially understood reading material will, by the simple fact of quantitative exposure, bring about a qualitative change, namely, the acquisition of both specialized terminology and general vocabulary. For many students, however, reading in context does not provide sufficient clarification and reinforcement to lead from contextual comprehension to the acquisition of general vocabulary. Furthermore, in certain cases it may even become pointless to teach reading skills and strategies: they cannot develop when the texts to be read are loaded with words totally unfamiliar to the reader. It seems to be obvious, therefore, that there is an urgent need for a general vocabulary building programme.

It has been acknowledged by a number of researchers (Levenston, 1979; Palmberg, 1987) that the acquisition of vocabulary is governed by the 
same factors as the learning of other aspects of the Foreign Language (FL) or the learning of the Foreign Language in general.

Accordingly, factors such as linguistic features (of the target language, of the learner's first language (L1) and of other languages known to the learner), learner factors (demographic variables, language background, learning strategies, attitudes) and situational factors (presentation, practice, testing) have to be considered in the development of a vocabulary programme.

The research project presented here concentrated on the method of vocabulary presentation which is considered an integral part of the initial stage of word learning. The underlying assumption was that the different methods of vocabulary presentation combine differently with learner factors (learner's gender, L1, parents' language, knowledge of other languages, individual learning strategies, attitudes, motivation, etc.) in vocabulary retention.

The question that arose as obvious was if there would be a substantial difference between the short-term and long-term retention of the same vocabulary with respect to different methods of vocabulary presentation as well as to the various learner factors.

The following research questions were specifically asked:

1. To what extent are the differences in the retention of vocabulary related to different methods of vocabulary presentation?

2. How do different methods of vocabulary presentation combine with different learner factors?

3. To what extent are the findings related to questions 1 and 2 valid with regard to the short-term and the long-term retention of the same vocabulary?

\section{Method}

Sixty Israeli first year university students majoring in social sciences and taking advanced level EFL courses were the subjects of the project. The subjects answered questions on a preliminary questionnaire which was meant to provide information on learner factors (personality variables, language background, word-processing habits and language attitudes). This information was coded according to the nature of the questions: a) ordinally (yes/no); (b) nominally (on a five point scale, ranging from "very much" to "very little"). (See Appendix 1). The information about the subjects' word-processing habits was grouped into the following categories:

- repeating orally

- writing down

- grouping into categories 
- finding associations

- trying to remember the image of the word

- skipping the unknown word

Any other category used by individual subjects and not listed above was coded as "other". (See Appendix 3).

Sixty-five words (with average frequencies 1 or 2) were randomly chosen from the Thorndike Frequency List (Thorndike and Lorge, 1963). The words were divided into 7 groups. Every week, for 7 consecutive weeks, 10 words were presented to the subjects (except for the 7th week, when only 5 words were presented).

The method of presentation was different in each of the sessions. The ordering of presentation as well as the ordering of words in each presentation was done on a random basis. This produced the following order: Word and its Translation, Visual Presentation (in print); Word with Picture; Word in Context, Auditory Presentation (recorded); Word and its Definition, Visual Presentation; Word and its Translation, Auditory Presentation; Word in Context, Visual Presentation; Three-fold Computer presentation (word and its definition, word presented in analogy, word in context). (See Appendix 3)

Subjects were given time to study the words and were instructed to simultaneously report in writing how they tried to remember them (Cohen, 1984). They were also asked to hand in their Learning Sheets on which they made notes, while trying to remember the word (each one in his/her own way of learning). This supplemented the Verbal Protocols, providing information about the learning process. The information derived from the subjects' Verbal Protocols and Learning Sheets was grouped into the following learning strategies: "translating the word", "finding familiar sounds (phonological association), "finding familiar elements in spelling and word form" (orthographic and morphological association), "repeating orally and in writing, "grouping the words according to concepts" (conceptual association), "changing the provided context". (See Appendix 2).

After each presentation the subjects were tested on the words they had just learned (short-term retention tests). The order in which the words were presented in the test and the test method-recall, translation from L1 to FL-were different from those of the presentation for learning. All the tests were paper-pencil tests.

To test long-term retention of the studied vocabulary, three months after the last learning session, a test composed of a random choice of $15 \%$ of the words was given to the learners. The method of testing was the same as the method of short term retention tests.

The test results and the information gained from the Preliminary Questionnaire, the Verbal Protocols and the Learning Sheets were analyzed. The following statistical operations were performed: Descriptive statistics, 
(means, standard deviations and Pearson correlations), Duncans' Multiple Range Test, Cronbach Reliability Test.

\section{Results and Discussion}

The scores on the 7 short-term retention tests showed a considerable range (from 89\%-57\%) (See Table 1). The highest scores on the short-term retention tests were obtained on the tests of visual presentation.

Table 1

Means and S.D.'s of Test-Scores in Percentages

(presented in rank-order)

\begin{tabular}{clcc} 
& \multicolumn{1}{c}{ Test } & X & S.D. \\
Short term & -Word w/picture & 88.93 & 14.36 \\
tests & - Word and its translation (visual) & 88.67 & 16.41 \\
& -Word and its Definition (visual) & 78.00 & 21.38 \\
& - Word in Context (visual) & 69.30 & 23.21 \\
& - Word in Context (auditory) & 65.00 & 21.13 \\
& - Three-fold, Computer Presentation & 62.71 & 25.31 \\
& -Word and its Translation (auditory) & 57.29 & 18.83
\end{tabular}

Long term

$\begin{array}{llll}\text { test } & - \text { Final test } & 39.50 & 12.41\end{array}$

The relatively high scores as well as the good results of visually presented words in the final test can be interpreted by a number of factors.

In the case of visual presentation, whether in printed form or with a picture, the learners are apparently assisted by their long-standing visual learning habits (as testified by their answers to the related specific question in the Preliminary Questionnaire). It also seems to be easier to recall the visual image of a word even in the context of long-term memory. The task of recalling an auditory image appears to require more concentration on the message as a whole. In the listening process the individual unfamiliar lexical item may be ignored and yet, the general message retrieved (Reves and Levine, 1988). Our data show that learners chose visual strategies even when the word was presented auditorily: their learning sheets were covered with scribbled word lists of the words they heard. Another possible explanation for the more successful recall of words presented visually may be based on the assumption that there is more danger for the learners to mishear a word, than for them to misread it. Since the tests were all paper-pencil tests (also when the presentation was auditory), the learners may have failed to recognize the written form of the words for which they had to provide an equivalent in Hebrew on the test. Apparently, they could not build the link between the sound combinations which they tried to remember and the meaning they had to provide. In addition, the spelling 
of the words-when presented visually, in print-may have served as a clue in the attempt to find a helpful association, even though sometimes it was based on false cognates (ransack-was translated by a number of students as merotz chipusim, which in Hebrew means run and search).

Within the visual presentations, the contextualized presentation was less effective than the decontextualized one (word and its definition or translation). This suggests that context may be helpful when the task is comprehension or deducing word meaning from context; it may, however, turn into an obstacle when the task is retention. The context may blur the focus on the word itself, the word may be lost in the flow of language i.e. in the longer discourse unit, sentence, paragraph, etc.

The results on the threefold presentation of words with the help of the computer were surprisingly low. It was assumed that providing more various exposure to the word, i.e. combining definitional, analogous and contextual information, would facilitate learning and improve retention. The assumption was supported by the suggestion that "the harder one works to process stimuli, the better one's retention". (Blachowicz, 1985).

It was also assumed that the use of the keyboard in combination with the computer screen would help to keep the student actively involved in the process of word learning and thus yield good results. These suppositions were corroborated by the students' enthusiasm for the novelty of the method. However, the feedback-remarks received from the subjects after the test (all of which related to technicalities of the presentation) provide, in a way, explanations as to why the actual achievement on this test was low: (a) it was harder for the subject to read the words and sentences from the screen than from the page on the table in front of them (they did not have enough practice in handling the computer); (b) the three-fold presentation of the same word confused them instead of helping them; (c) the subjects would have preferred to see the complete list of the words to be learned on top of the screen instead of being presented with one word at a time (this seems to have imposed on them one specific learning strategy, which many of them may not have employed had the choice been theirs).

The immediate negative feedback-as well as the low scores on this test-should not result in the rejection of a multiple method of vocabulary presentation, or even in the discarding of the use of the computer for vocabulary learning. The analysis of the test results showed significant relationships between this method and the learners' positive attitude to language learning.

Moderate, statistically significant correlations were found between test results and two common aspects within the different methods of vocabulary presentation: a) when the mode of presentation was the same (either visual or auditory); b) when the amount of contextualization was the same (either only a definition/translation or a broader context). Thus, the scores 
on the two tests where the mode of presentation was visual showed a correlation of .44 and, similarly, scores on the two tests where the mode of presentation was auditory showed a correlation of .44. The test scores on both presentations of Word in Context (visual and auditory) yielded a correlation of .43 . In other words, test results "clustered" around two very specific aspects in the presentation of vocabulary: mode and context. These findings support the notion that different learning patterns or cognitive styles, i.e. the visual and the auditory and the contextually associative, predict clearly identifiable ways of the learning and retention of vocabulary.

The test scores on the long-term retention tests were much lower than those on the short-term retention tests. (See Table 1). It was noted by Stevick (1982) that whether the material makes it from short-term memory to long-term or permanent memory, is largely affected by three factors: recency, frequency and intensity of exposure. Since the subjects in our experiment did not have any further exposure to or rehearsal of the items between the presentation sessions and the long-term retention test, neither the recency nor the frequency factor could have contributed to the retention of the vocabulary in the long-term memory. The intensity factor-vividness of exposure, importance and/or emotional depth at which the material to be remembered affects the learner (Stevick, 1982) - was not strong enough to ensure permanent memory and yield good results on the long-term retention test.

The results on three tests of visually presented vocabulary were related to the subjects' L1 and/or parents' language. This is probably due to the fact that some words in the test were etymologically related to the languages in the subjects' language background. The evocation of the cognate in the learner's linguistic memory was facilitated by the visual presentation of the word.

The tests on three visual presentations of vocabulary yielded significant relationships with the learners' gender. The hypothesized superiority of female learners in the field of verbal tasks (Maccoby and Jacklin, 1974) might tentatively be ascribed to a different level of field-independence: female learners' visual observation of details may be more acute and thus their ability to remember visually presented material might be better.

The relationship found between the learner's memory and auditorily presented vocabulary allows us to hypothesize that memory is consciously drawn upon when there is no visual support provided to the task of learning. A parallel phenomenon-of consciously drawing on additional resources-was observed in the relationship between the results of the long-term retention test and learner factors of linguistic nature: L1, parents' language and additional languages known by the subjects. The learners could not reproduce most of the meanings of the words on the test 
because the immediate source of their long-term memory failed them. It is conceivable that in an effort to avoid failure they may have drawn on other linguistic resources.

Relationships between test results and learning strategies were mostly derived from tests of auditory presentation. This is of special interest since the scores on the tests of visual presentations of vocabulary were much higher than those of auditory presentations. Our tentative suggestion is that when the learning task is hard, as in the learning of auditorily presented vocabulary, the strategies are activated to a greater extent. This seems to be similar to the case of communicative strategies, which are mobilized when the need for bridging the gap in communication arises (Canale \& Swain, 1980).

The relationship discovered between the self-reported difficulty in comprehension caused by an unknown word and the test-results of auditorily presented vocabulary was negative. This suggests the following interpretation: when the learner has to cope with auditorily presented material, the attention is drawn to the message as a whole, so that less importance is attached to the individual items. (See Reves and Levine, 1988). Thus the unknown individual words do not seem to be an obstacle to comprehension.

Information on the subjects' vocabulary processing strategies was obtained from two sources: a) the preliminary questionnaire and b) the subjects' authentic Verbal Protocols and Learning Sheets. The subjects self-reported vocabulary processing strategies (as reflected in the preliminary questionnaire) were noticeably different from the strategies evidenced by their verbal protocols and learning sheets.

The word-processing reported on the questionnaire and the strategies reflected in the protocols refer to the use of examples, dictionary, help from another language, etc. The learning sheets indicate, however, that the subject tried to learn the word by making up various word lists, changing the context, as well as with the help of linguistic and conceptual associations. The words were copied a number of times, grouped sometimes in alphabetical order, sometimes in a decreasingly selective order (each time excluding the words they felt they had mastered), sometimes by employing their own, completely different order of words. This information is worth noting: the words for the presentation were chosen on a random basis, thus no attention was paid to their clustering. Yet, students used "clustering" as their learning strategy. This might suggest that an organized presentation of words is probably more effective than a random presentation.

Furthermore, judging by the prevalence of repetition as a word-learning strategy among the subjects in our experiment, the importance of this method seems to be beyond doubt. 
Another observable strategy was the change of context. In cases where the word was presented in context, the learners often inserted a more frequently used word, familiar to them, as a synonym to the word they had to learn (e.g. ignominy $=$ shame; rancor $=$ anger; indigenous $=$ familiar).

The associations used by the learners as evidenced by their Learning Sheets were of different types: (a) based on sound similarity (e.g. gossamer/gossip; fritter/"freier"*; mongrel/mango), (b) based on orthographic analogy e.g. mitigate/gate; penury/pen; abase/"abba"**; (c) based on false morphological associations e.g. nebulous/less; rectify/rein the sense of repetition; (d) based on conceptual associations e.g. rancor-"kor"***, ransack/ = ran/merotz chipusim ${ }^{* * * *}$; hurtle $=$ injure (by throwing you hurt or injure someone).

There seems to be no way of knowing whether what the subjects report is the result of their conscious awareness or momentary observation of their own strategies of learning, rather than a statement based on what they think might satisfy the experimenter. Hence, the subjects verbal selfreports, although, without doubt, a valuable source of information, should be used and interpreted with utmost caution.

An obvious relationship was found between methods of presentation on the one hand and motivation and attitudes on the other. The conventional task of learning listed vocabulary items - which even shows in their learning sheets as an ingrained habit-was associated by the learners with their previous experience of failure in FL learning; hence the relationship with the self-reported difficulty in language learning, which can easily be interpreted as a negative attitude. The novelty of the method of the threefold computer presentation, on the other hand, seems to be attractive and motivating; it does not remind the learner of experiences of previous failure, and is therefore related to positive attitudes. Even the technical difficulties involved in this-still very experimental-method, though reported by the students, do not change their positive attitudes.

\section{Conclusions}

In conclusion, relating to the research questions posed at the outset of the study, the data obtained from the project indicate the following findings:

\footnotetext{
* Hebrew slang for "sucker"

** Hebrew word for "father"

*** Hebrew word for "cold": the association of unpleasantness

**** Hebrew word for "run and search"
} 
a. The method of presenting new vocabulary to students of professional, academic reading courses undoubtedly leads to different degrees of vocabulary retention. The retention of vocabulary seems to be related to the learner's general learning patterns or cognitive styles of visuality, auditiveness or contextual association. Of these, visual presentation appears to lead to higher levels of retention and should thus be recommended.

b. The fact that various learner factors (language background, gender, memory, word-learning strategies, language attitudes) combined differently with a number of methods of vocabulary presentation supports the assumption that the processing of new vocabulary is both individual and manifold. It seems to be advisable not to impose any one specific method on the learner. Vocabulary should be presented by a variety of techniques: methods should be varied and combined according to the learner's individual needs and preferences. The positive attitudes related to the computer presentation may suggest that vocabulary presentation should capitalize on the attraction of novelty.

c. Most of the findings regarding the short-term retention of vocabulary do not seem to be validly applicable to the long-term retention of vocabulary. This was probably an effect of the overall failure of the long-term retention hypothesis. As suggested in the discussion, no long-term retention achievement can be expected as a result of one single exposure to and no recycling of vocabulary items. There were, however, different relationships inn the case of long-term retention which did not show in the short-term retention context. A variety of linguistic associations used as a resource by the learner were an interesting finding regarding long-term retention. In vocabulary teaching frequent and numerous recycling should probably be emphasized.

Vocabulary is cumulatively gaining ground among the declared aims of the instruction of reading comprehension. Further research is obviously needed to investigate the effect of different methods of teaching and learning vocabulary.

\section{APPENDIX 1 \\ QUESTIONNAIRE}

Surname

Name

Gender

1. What is your L1?

2. What is your parents' L1?

3. Do you know other languages?

Language 

(4)
(3)
(2)

Speaking Very good Good Satisf. Poor Very poor

Reading Very good Good Satisf. Poor Very poor

Writing Very good Good Satisf. Poor Very poor

Language

Speaking Very good Good Satisf. Poor Very poor

Reading Very good Good Satisf. Poor Very poor

Writing Very good Good Satisf. Poor Very poor

4. Do you consider your memory good/poor?

5. When do you remember things better: when you hear them/when you see them?

6. Do you remember better general things/details?

7. When you learn words by heart, do you pronounce them? YES/NO

8. Suppose that you come across unknown words while reading or listening. Does it interfere with your general comprehension of the context? YES/NO

9. When you do not understand a word, do you look for help in another language you know?

always often sometimes rarely never

10. When you use an English-English dictionary, do you read the definition only or do you also read the examples?

always often sometimes rarely never

11 . When you use the dictionary do you pronounce the words aloud? always often sometimes rarely never

12. When you learn new words in a FL, do you try to connect the new words to words you already know?

always often sometimes rarely never

13. When you learn new words in a FL, do you make up word lists and practise them?

always often sometimes rarely never

14. When you learn new words in a FL, do you try to find associations between them and

a) the words in your L1

always often sometimes rarely never

b) other words in the same FL?

always often sometimes rarely never

c) pictures or movements (gestures)?

always often sometimes rarely never

15. Suppose you have to learn something by heart (rules, dates, formulas, names, etc.). Describe how you do it, step by step.

16. Is foreign language learning in general very important/important/ not particularly important/unimportant/ not important at all?

17. Is the learning of English as a foreign language very important/ 
important/not particularly important/unimportant/not important at all?

18. Did you have difficulties learning English in the past? List them.

19. Are you ready to make special efforts to learn a FL well? YES/ NO

\section{APPENDIX 2}

List of Variables and Categories Derived from the Preliminary Questionnaire

(Personality Variables, Language Background, Word-processing Habits and Language Attitudes)

Gender

Mother Tongue

Parents' Language

Additional Languages known by subject

Memory (e.g. type, characterstics)

Word-processing habits (e.g. skipping the unknown word, resortng to $\mathrm{L} 1$ associations)

Language attitudes (e.g. the importance of language learning, readiness to make efforts, difficulties in language learning)

\section{List of Word-Learning Strategies Derived from Verbal Protocols and Learning Sheets}

Translation of word

Repetition of word (oral or written)

Phonological association

Morphological association

Orthographical association

Conceptual association

Providing additional context

\section{APPENDIX 3}

1. Word and its translation, visual presentation

1. guileless

2. ignominy

3. eschew

4. aspersion

5. facetious

6. insipid

7. glutton

8. hurtle

9. desultory

10. carnage 
2. Word and its definition, visual presentation

$\begin{array}{ll}\text { 1. abase } & - \text { humiliate } \\ \text { 2. crevice } & - \text { narrow opening } \\ \text { 3. fritter } & - \text { waste } \\ \text { 4. penury } & - \text { poverty } \\ \text { 5. frisky } & - \text { lively } \\ \text { 6. splutter } & - \text { speak quickly } \\ \text { 7. lubricate } & - \text { oil } \\ \text { 8. larceny } & - \text { stealing } \\ \text { 9. waive } & - \text { not to insist } \\ \text { 10. mitigate } & - \text { make less painful }\end{array}$

3. Word in context, visual presentation

1. In one year he squandered his entire fortune on boats, parties, trips abroad. No wonder, he is poor now.

2. The man pilfered money from his company for several years before the losses were discovered.

3. Oranges are indigenous to the southern areas of the United States but are foreign to the northern areas.

4. Ignominy was brought upon the family when their son was arrested and put to prison. The town paper printed the story and soon many friends stopped talking to them.

5. I am surprised you are talking about the details instead of concentrating on the most salient points of the speech.

6. He made a lot of money when he sold his house. The deal was lucrative.

7. He rummaged among the books till he found his old diary.

8. Mother and son avoided an open rupture by never mentioning their differences.

9. He was convinced his friend was guilty of his misfortune and did not even try to hide his rancor against him.

10. It is the law of nature that the strong rule; everyone recognizes this law in fact, though repudiates it in theory.

\section{Word with picture}

conifer

vial

bight

forelock

harpoon

moat

spigot

pinnacle

dice

muzzle 
5. Word and its translation, auditory presentation

jowl

effervescent

gossamer

petulant

defunct

rowdy

boatswain

incipient

encrust

declivity

\section{Word in context, auditory presentation}

The sun could hardly be seen through the musk of clouds.

She simpered as if she hadn't understood his remark; but this smile of hers did not bother him.

The conflict developed into a hectic tussle between the two opponents.

The ostensible purpose of his trip was to visit his aunt; yet, all his friends knew that this was only an excuse.

Their wanton cruelty upset everyone who witnessed the attack.

They were all reliable stalwarts of the new Minister; he knew he could count on their loyalty.

His old quips still make everybody laugh.

I feel I vacillate between admiration and pity for him.

The windows were thick with grime: no one could see through them. They needed a good cleaning.

The eggs frizzled in the pan: they go all burnt; we could hardly eat them.

7. Threefold, computer presentation

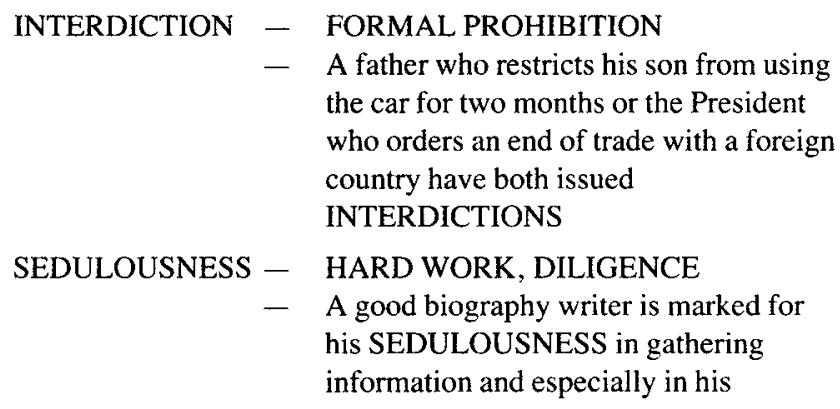
the car for two months or the President who orders an end of trade with a foreign country have both issued INTERDICTIONS
SEDULOUSNESS - HARD WORK, DILIGENCE
- A good biography writer is marked for his SEDULOUSNESS in gathering information and especially in his




\begin{tabular}{|c|c|c|}
\hline & - & $\begin{array}{l}\text { diligence in worting it out. } \\
\text { ATRACTIVENESS is to APPEAR- } \\
\text { ANCE as SEDULOUSNESS is to } \\
\text { WORKMANSHIP }\end{array}$ \\
\hline SINUOUS & - & $\begin{array}{l}\text { FULL OF CURVES, TWISTING } \\
\text { The red lava flowed down the } \\
\text { mountainside in a SINUOUS ribbon, } \\
\text { like a snake moving towards its prey. } \\
\text { SINCERE is to ATTITUDE as } \\
\text { SINUOUS is to MOVEMENT. }\end{array}$ \\
\hline PERFIDIOUS & - & $\begin{array}{l}\text { FAITHLESS } \\
\text { He was one of the most trusted members } \\
\text { of the party but when they learned about } \\
\text { his PERFIDIOUS acts, they understood } \\
\text { that he was disloyal. } \\
\text { DECEPTIVE is to HONESTY as } \\
\text { PERFIDIOUS is to LOYALTY. }\end{array}$ \\
\hline IMPRECATION & - & $\begin{array}{l}\text { CURSE } \\
\text { The driver accepted the ticket from the } \\
\text { police, uttering IMPRECATIONS } \\
\text { under his breath as he drove away. } \\
\text { COMPLEMENT is to APPRECIATION } \\
\text { as IMPRECATION is to HOSTILITY. }\end{array}$ \\
\hline
\end{tabular}

\section{REFERENCES}

Blachowicz, C., (1985). Vocabulary development and reading. The Reading Teacher, 38, 876-881.

Canale, M. \& M. Swain, (1980). Theoretical bases of communicative approaches to second language teaching and testing. Applied Linguistics, 1, 1-38.

Cohen, A. D. (1984). Studying second language learning strategies: How do we get the information? Applied Linguistics, 5(2): 101-112.

Levenston, E. (1979). Second language vocabulary acquisition: Issues and problems. Interlanguage Studies Bulletin, 4, 147-60.

Maccoby, E. E., \& C. N. Jacklin, (1974). The Psychology of Sex Differences, Stanford, California, Stanford University Press. 74-75.

Palmberg, R. (1987). Patterns of vocabulary development in foreign language learners. Studies in Second Language Acquisition, 9, 201-220.

Reves, T. \& A. Levine, (1988). The FL receptive skills: Same or different? System, Pergamon Press, Oxford, vol. 16, N 3, 327-336.

Stevick, E. (1982). Teaching and Learning Languages, Cambridge, Cambridge University Press, 30-31. 
Thorndike, E. L. \& I. Lorge, (1963). The Teacher's Word Book of 30,000 Words, New York: Columbia University.

\section{THE AUTHORS}

Adina Levine holds a Ph.D. degree in Linguistics from the Hebrew University, Jerusalem. She is a senior teacher in the English Department at Bar-Ilan University, where she teaches advanced grammar and English for Academic Purposes. Her present research interests focus on the skills and strategies of reading comprehension. She is co-author of two books on reading comprehension for university students.

Thea Reves holds a Ph.D. degree from the the Hebrew University, Jerusalem. She is a senior teacher of EFL at Bar-Ilan University, and the Inspector of TEFL, Ministry of Education. She is in charge of curriculum planning, teacher training, and the construction of national exams in EFL. She is the co-author of an oral proficiency test-battery introduced as the national school-leaving exam. 